\title{
Semi-classical Limit in a Semiconductor Superlattice
}

\author{
PHILIPPE BECHOUCHE* \\ Univ. Nice, Lab. J. A. Dieudonné URM 6621 du CNRS, Parc Valrose, F-06108 Nice, France
}

(Received 13 August 1997; In final form 1 December 1998)

\begin{abstract}
In this paper, we perform a mathematical study of a semiconductor superlattice. Since the thickness of the layers is very small, quantization plays an important role. The modelling is therefore given by a Schrödinger equation with a periodic potential. The scaled lattice thickness is denoted by a small parameter $\varepsilon$ which is of the same order of magnitude as the Planck constant. When this parameter tends to zero, i.e., the semi-classical limit, we obtain classical transport of the charge carriers described by a Vlasov equation.
\end{abstract}

Keywords: Semiconductor, superlattice, quantum transport, Schrödinger equation, semi-classical limit

\section{INTRODUCTION}

Superlattices technology represent a modern technology used also in the design of semiconductors. In order to gain insight in the complex physical behaviour of these devices it is sometimes necessary to have very precise models which give a description on the atomic level. In this work we start with such a fundamental model that takes in account the quantum effects and investigate its semi-classical approximation in a mathematically rigorous way.

A superlattice can be described as material which consists of a stack of layers, those will be of two kinds: a layer $A$ and a layer $B$ as it is shown on Figure 1.
This is the model of $\mathrm{Si} / \mathrm{Ge}, \mathrm{GaAs} / \mathrm{AlAs}$ or InAsSb/InSb superlattices for instance.

In the first part of this paper, we give a quantum description of such superlattices describing structures which are periodic in one direction $x_{1}$ but whose properties vary slowly in other directions $x_{2}, x_{3}$. This means that in this model we regard layers which have a slowly varying doping profile.

The motion of a charged particle in a stratified medium can be described by the Schrödinger equation:

$$
\begin{aligned}
i \hbar \frac{\partial \Psi}{\partial T} & =-\frac{\hbar^{2}}{2 m} \Delta_{X} \Psi(X, T)+q V(X) \Psi(X, T) \\
X & =\left(X_{1}, X_{2}, X_{3}\right) \in \mathbb{R}^{3}, T \in \mathbb{R}
\end{aligned}
$$

\footnotetext{
*e-mail: phbe@math.unice.fr
} 


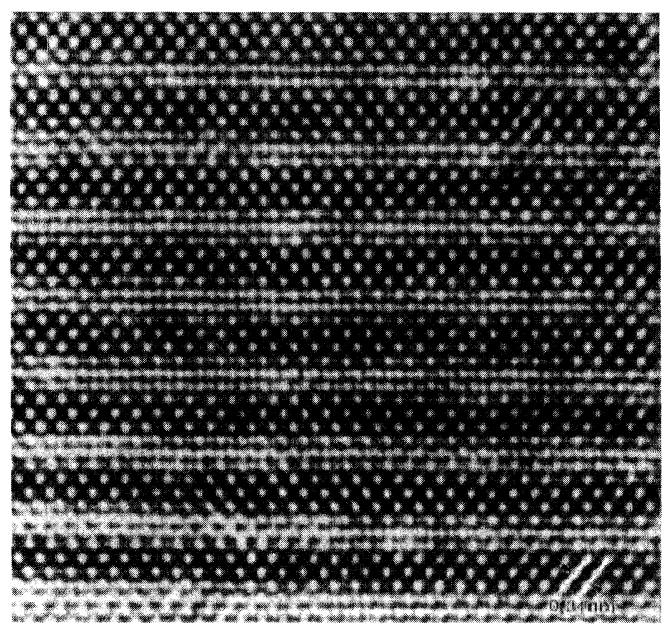

FIGURE 1 Compositional and structural characterization of a $\mathrm{Si} / \mathrm{Ge}$ superlattice and heterostructures by high resolution transmission electron microscopy.

where $\hbar$ is the Planck constant, $q$ the charge of the particle and $m$ its mass. In our "stratified medium" the crystal is periodic with period $\lambda$ in the direction $X_{1}$ such that the potential $V$ verifies:

$$
V\left(X_{1}+\lambda \gamma, X_{2}, X_{3}\right)=V\left(X_{1}, X_{2}, X_{3}\right), \quad \gamma \in \mathbb{Z} .
$$

We will also assume that the potential is bounded, i.e.,

$$
V \in L^{\infty}\left(\mathbb{R}^{3}\right)
$$

Let us define the quantum scale:

$-\lambda$ is the length of the period of the medium in the direction $X_{1}$

- $\tau$ is its time unit such that we have (e.g. [10])

$$
\tau=\frac{m \lambda^{2}}{h}
$$

Now if we denote by $L$ and $T_{0}$ the macroscopic length of the crystal, we can define a scaled potential by:

$$
q V(X)=\frac{m \lambda^{2}}{\tau^{2}} v\left(\frac{X_{1}}{\lambda}, \frac{X_{2}}{L}, \frac{X_{3}}{L}\right)
$$

Let us introduce new variables $x, t, \varepsilon$ :

$$
x=\frac{X}{L}, t=\frac{T}{T_{0}}, \varepsilon=\frac{\lambda}{L}
$$

We now scale the wave function.

We recall that $\Psi$ has to be normalized in such a way that:

$$
\int_{\mathbb{R}^{3}}|\Psi(X, T)|^{2} d X=1
$$

So we put:

$$
\psi(x, t)=L^{3 / 2} \Psi\left(L x, T_{0} t\right)
$$

We remark that $\psi$ is also normalized.

Let us choose $T_{0}$ and $L$ such that:

$$
\frac{\hbar}{T_{0}} \cdot \frac{\tau^{2}}{m \lambda^{2}}=\varepsilon \quad \text { and } \quad \frac{\hbar^{2}}{2 m L^{2}} \cdot \frac{\tau^{2}}{m \lambda^{2}}=\frac{\varepsilon^{2}}{2}
$$

With this scaling Eq. (1.1) becomes:

$$
\begin{array}{r}
i \varepsilon \partial_{t} \psi=-\frac{\varepsilon^{2}}{2} \Delta_{x} \psi(x, t)+v\left(\frac{x_{1}}{\varepsilon}, x_{2}, x_{3}\right) \psi(x, t) \\
x \in \mathbb{R}^{3}, t \in \mathbb{R}
\end{array}
$$

We want to study rigorously the semi-classical limit of 1.7 when $\varepsilon$ goes to zero. In particular we are concerned with the limit behavior of the density:

$$
n_{\varepsilon}(x, t)=|\psi(x, t)|^{2}
$$

Semi-classical limits of Schrödinger operators have been intensively studied. In the case of perturbed periodic potential we refer to [2]. In this works the limit behaviour of the Schrödinger operator is given. Our purpose is to study the limit behaviour of the particle dynamics. This problem has been already been investigated in the case of: $-v$ periodic $[8,6]-v \in C^{1}[4,7,6]-v+u$ with $v$ periodic and $u$ a small perturbation $[9,1]$. The result in the periodic case is that the limit concentration is given by the sum of the concentrations $n_{p}(x, t) p=1, \ldots, \infty$ 
corresponding to the eigenvalue problems e.g. [8].

$$
\begin{aligned}
- & \frac{\varepsilon^{2}}{2} \frac{d^{2}}{d x_{1}^{2}} \psi_{p}\left(x_{1}, k_{1}\right)+v\left(\frac{x_{1}}{\varepsilon}\right) \psi_{p}\left(x_{1}, k_{1}\right) \\
& =E_{p}(k) \psi_{p}\left(x_{1}, k_{1}\right) \psi_{p}\left(x_{1}+\varepsilon \gamma, k_{1}\right) \\
& =\mathrm{e}^{i \psi k_{1} \gamma} \psi_{p}\left(x_{1}, k_{1}\right)
\end{aligned}
$$

and the concentration are given by:

$$
n_{p}(x, t)=\int_{\mathbb{R}^{3}} f_{p}\left(x, k_{1}, t\right) d k_{1}
$$

where the non negative distribution $f_{p}$ solves the transport equations.

$$
\partial_{t} f_{p}\left(x, k_{1}, t\right)+\partial_{k_{1}} E_{P}\left(k_{1}\right) \cdot \partial_{x_{1}} f_{p}\left(x, k_{1}, t\right)=0 .
$$

In this work the main tools are the Wigner transform of wave functions and the Bloch decomposition of Schrödinger operators.

The Bloch decomposition e.g. $[12,4,11]$ is the projection of wave functions on generalized eigenspaces, the Floquet spaces. In our framework they depend on $x_{2}, x_{3}$. To each eigenspace $V_{p}\left(x_{2}, x_{3}\right)$ corresponds an energy band $E_{p}\left(k_{1}, x_{2}, x_{3}\right)$ and the projector $\Pi_{p}\left(x_{2}, x_{3}\right)$. The main difficulty is that the projectors $\Pi_{p}\left(x_{2}, x_{3}\right)$ do not commute with $\left(-\varepsilon^{2} / 2\right) \Delta_{x_{2}, x_{3}}$.

We overcome this difficulty by using asymptotic expansions of Wigner transforms given in [6].

In the following of this paper, we will make the assumption that the energy Bands don't cross. In fact it is possible to choose the eigenvectors of the Bloch decomposition such that the energy Bands do not cross. Such an choice can always been done for a Bloch decomposition in one dimension, which is the case here, but it has been proved that it is false in higher dimensions (e.g. [5, 3]).

The result is that the distribution function solves the transport equation:

$$
\begin{aligned}
\frac{\partial}{\partial t} f_{p}(x, k, t)= & \left(\begin{array}{c}
\partial_{k_{1}} E_{p}\left(k_{1}, x_{2}, x_{3}\right) \\
k_{2} \\
k_{3}
\end{array}\right) \cdot \nabla_{x} f_{p}(x, k, t) \\
& +\nabla_{x_{2}, x_{3}} E_{p}\left(k_{1}, x_{2}, x_{3}\right) \cdot \nabla_{k_{2}, k_{3}} f_{p}(x, k, t)
\end{aligned}
$$

and the limit concentration $n$ is given by:

$$
n(x, t)=\sum_{p} \int_{[-\pi, \pi] \times \mathbb{R}^{2}} f_{p}(x, k, t) \frac{d k_{1}}{2 \pi} d k_{2} d k_{3}
$$

We remark in Eq. (1.9) that the band velocity in the first variable

$$
\partial_{k_{1}} E_{p}\left(k_{1}, x_{2}, x_{3}\right)
$$

depends on the two parameters $x_{2}, x_{3}$ and that in the other variable the velocity corresponds to free transport.

This paper is organized as follows: In the next section we introduce the Bloch decomposition of the wave operators but only in the $x_{1}$-direction and examine the $x_{2}, x_{3}$ dependence of the projectors and the energy bands. Then we derive a Wigner equation by using Wigner series in the direction $x_{1}$ and Wigner transforms in the directions $x_{2}, x_{3}$. In the last section we pass to the semi-classical limit and give the main results.

The main results concerning Bloch waves are used in $[2,4,6]$ and those concerning Wigner transforms and series in $[7,4,6]$.

In this paper we will assume that the initial data is $\varepsilon$-ocillating; more precisely we assume:

$$
\text { for }|\alpha| \leq 4 \quad \int_{\mathbb{R}^{3}}\left|D^{\alpha} \psi_{I}(x)\right|^{2} d x \leq \frac{C}{\varepsilon^{2|\alpha|}} \quad \alpha \in \mathbb{N}^{3}
$$

Remark 1.1 As in [6], this assumption can be weaken to the usual condition of $\varepsilon$-ocillation of [4].

Since the energy of the system reads

$$
E(t)=\frac{\varepsilon^{2}}{2} \int_{\mathbb{R}^{3}}|\nabla \psi|^{2} d x+\int V\left(\frac{x_{1}}{\varepsilon}, x_{2}, x_{3}\right)|\psi|^{2}
$$

this implies that

$$
\int_{\mathbb{R}^{3}}|\nabla \psi(x, t)|^{2} d x \leq \frac{C}{\varepsilon^{2}}
$$




\section{BLOCH DECOMPOSITION}

In this section we will recall classical results about Bloch decomposition. But here we only make onedimensional $\left(x_{1}\right)$ Bloch decomposition and then analyse the regularity of the eigenvalues and of the projectors w.r.t. $x_{2}$ and $x_{3}$. We want to "diagonalize" the Hamiltonian

$$
H \psi=-\frac{\varepsilon^{2}}{2} \cdot \frac{\partial^{2} \psi}{\partial x_{1}^{2}}+V\left(\frac{x_{1}}{\varepsilon}, x_{2}, x_{3}\right) \psi
$$

If we consider the microscopic scale $(e . g .[10,1])$ in the first variable,

$$
\psi_{1}\left(y_{1}, x_{2}, x_{3}\right)=\varepsilon^{1 / 2} \psi_{2}\left(\varepsilon y_{1}, x_{2}, x_{3}\right)
$$

As in $[10,4]$ or $[6,1]$, we expand the function $\psi_{1} \in L^{2}\left(\mathbb{R}^{3}\right)$ into the Block waves

$$
\begin{gathered}
\phi\left(y_{1}, x_{2}, x_{3}, k\right)=\sum_{\gamma \in \mathbb{Z}} \psi_{1}\left(y_{1}-\gamma, x_{2}, x_{3}\right) \mathrm{e}^{i k_{1} \gamma}, \\
k \in \mathbb{R}
\end{gathered}
$$

\section{Properties of the Bloch Waves}

Property 2.1

$$
\begin{gathered}
\Psi_{1}\left(y_{1}, x_{2}, x_{3}\right)=\int_{-\pi}^{\pi} \phi\left(y_{1}, x_{2}, x_{3}, k_{1}\right) \frac{d k_{1}}{2 \pi}, \\
\|\phi\|_{L^{2}\left([0,1] \times \mathbb{R}^{2} \times[-\pi, \pi]\right)}=\left\|\psi_{1}\right\|_{L^{2}\left(\mathbb{R}^{3}\right)}
\end{gathered}
$$

Property $2.2 \psi$ is $k$-periodic i.e.,

$$
\phi\left(y_{1}+\gamma, x_{2}, x_{3}, k_{1}\right)=\mathrm{e}^{i k_{1} \gamma} \phi\left(y_{1}, x_{2}, x_{3}, k_{1}\right)
$$

Let $H$ be the Hamiltonian defined on

$$
D(H)=\left\{\psi \in L^{2}\left(\mathbb{R}^{3}\right), \frac{\partial^{2}}{\partial y_{1}^{2}} \psi \in L^{2}\left(\mathbb{R}^{3}\right)\right\}
$$

Property 2.3 If $H_{k_{1}}$ is the Hamiltonian which acts on $k$-periodic functions i.e.,

$$
\begin{aligned}
& D\left(H_{k_{1}}\right) \\
& =\left\{\phi\left(y_{1}, x_{2}, x_{3}, k_{1}\right) \in L^{2}\left([0,1] \times \mathbb{R}^{2} \times[-\pi, \pi]\right),\right. \\
& \quad \frac{\partial^{2}}{\partial y_{1}^{2}} \phi\left(y_{1}, x_{2}, x_{3}, k_{1}\right) \in L^{2}([0,1] \\
& \left.\left.\quad \times \mathbb{R}^{2} \times[-\pi, \pi]\right)\right\}
\end{aligned}
$$

and

$$
\phi\left(y_{1}-\gamma, x_{2}, x_{3}, k_{1}\right)=\mathrm{e}^{i k_{1} \gamma} \phi\left(y_{1}, x_{2}, x_{3}, k_{1}\right)
$$

then e.g. $[12,11] H_{k_{1}}$ is a compact operator and diagonalizable. Moreover

$$
H \psi=\int_{-\pi}^{\pi} H_{k_{1}} \phi\left(., k_{1}\right) \frac{d k_{1}}{2 \pi}
$$

where $\phi$ is given by 2.2. Let us denote by $E_{1}\left(k_{1}, x_{2}, x_{3}\right), \ldots, E_{p}\left(k_{1}, x_{2}, x_{3}\right), \ldots$ the eigenvalues on $H_{k_{1}}$,

$$
\begin{aligned}
E_{1}\left(k_{1}, x_{2}, x_{3}\right) & \leq E_{2}\left(k_{1}, x_{2}, x_{3}\right) \\
& \leq \cdots \leq E_{p}\left(k_{1}, x_{2}, x_{3}\right) \leq \cdots
\end{aligned}
$$

and by $\phi_{p}\left(., k_{1}\right)=\phi_{p}\left(y_{1}, x_{2}, x_{3}, k_{1}\right)$ the associated eigenvectors. Then we have

THeOREM $2.1 c f .[11,12]$ There is a choice of the eigenvector family $\left\{\phi_{p}\left(., k_{1}\right)\right\}$ such that it is an Hilbert Basis of $L^{2}\left(\mathbb{R}^{3}\right)$ and such that the functions

$$
k_{1} \mapsto \phi_{p}\left(y_{1}, ., k_{1}\right) \overline{\phi_{p}}\left(z_{1}, ., k_{1}\right)
$$

are all analytical. The $\phi_{p}\left(., k_{1}\right)$ are $k_{1}$-periodic and $\phi_{p} \in L^{2}\left([0,1] \times \mathbb{R}^{2} \times[-\pi, \pi]\right)$.

Definition 2.2 Let us define now the Wannier functions:

$$
\psi_{p}\left(y_{1}, x_{2}, x_{3}\right)=\int_{-\pi}^{\pi} \phi_{p}\left(y_{1}, x_{2}, x_{3}, k_{1}\right) \frac{d k_{1}}{2 \pi}
$$

These functions are associated to the Bloch waves $\phi_{p}\left(., k_{1}\right)$. They also verify the Property 2.1 and the following properties. 


\section{Property 2.4}

$$
\phi_{p}\left(., x_{2}, x_{3}, k_{1}\right)=\sum_{\gamma \in \mathbb{Z}} \psi_{p}\left(\cdot-\gamma, x_{2}, x_{3}\right) \mathrm{e}^{i k_{1} \cdot \gamma} i e
$$

and $\psi_{p}\left(.-\gamma, x_{2}, x_{3}\right)$ are the Fourier coefficients of $\phi_{p}\left(., x_{2}, x_{3}, k_{1}\right)$.

\section{Property 2.5}

$$
\int_{\mathbb{R}^{3}}\left|\psi_{p}\left(y_{1}, x_{2}, x_{3}\right)\right|^{2} d y_{1} d x_{2} d x_{3}=1
$$

and

$$
\int_{\mathbb{R}} \psi_{p}\left(y_{1}, x_{2}, x_{3}\right) \overline{\psi_{p}}\left(y_{1}-\gamma, x_{2}, x_{3}\right) d y_{1}=0 \quad \gamma \neq 0
$$

Definition 2.3 Let us define the Floquet subspaces by

$$
\begin{array}{r}
V_{p}\left(x_{2}, x_{3}\right)=\left\{\psi \in L^{2}\left(\mathbb{R}^{3}\right), \psi\left(y_{1}, x_{2}, x_{3}\right)\right. \\
=\int_{-\pi}^{\pi} \alpha_{p}\left(k_{1}, x_{2}, x_{3}\right) \\
\phi_{p}\left(y_{1}, x_{2}, x_{3}, k_{1}\right) \frac{d k}{2 \pi} \\
\left.\alpha_{p} \in L^{2}\left([-\pi, \pi] \times \mathbb{R}^{2}\right)\right\}
\end{array}
$$

where the $\phi_{p}$ are the Bloch waves associated to $H_{k_{1}}$.

THEOREM $2.4 c f .[11,12]$

$$
L^{2}\left(\mathbb{R}^{3}\right)=\bigoplus_{p} V_{p}\left(x_{2}, x_{3}\right), \quad x_{2}, x_{3} \in \mathbb{R}
$$

Let $\hat{E}_{p}\left(\gamma, x_{2}, x_{3}\right), \gamma \in \Gamma$, be the Fourier coefficient of $E_{p}\left(k_{1}, x_{2}, x_{3}\right)$.We also have $V_{p}\left(x_{2}, x_{3}\right) \in D(H)$ and

$$
\begin{array}{r}
H(\psi)=\sum_{\gamma \in \mathbb{Z}} \hat{E}_{p}\left(\gamma, x_{2}, x_{3}\right) \psi\left(y_{1}+\gamma, x_{2}, x_{3}\right), \\
\psi \in V_{p}\left(x_{2}, x_{3}\right)
\end{array}
$$

moreover we have $H(\psi) \in V_{p}\left(x_{2}, x_{3}\right)$.
For the proof of Theorem 2.4 we refer to $[10,11,8,6]$.

Remark 2.5 The function $E_{p}\left(., x_{2}, x_{3}\right)$ is even. Therefore its Fourier coefficients satisfy

$$
\hat{E}_{p}\left(\gamma, x_{2}, x_{3}\right)=\hat{E}_{p}\left(-\gamma, x_{2}, x_{3}\right) \in \mathbb{R}
$$

Remark 2.6 The equation

$$
H_{k} \phi_{P}\left(y_{1}, x_{2}, x_{3}, k_{1}\right)=E_{p}\left(k_{1}, x_{2}, x_{3}\right) \phi_{P}\left(y_{1}, x_{2}, x_{3}, k_{1}\right)
$$

implies (by derivating in direction $x_{2}$, multiplying by $\overline{\phi_{p}}$ and integrating in $y_{1}$ ) that

$$
\begin{aligned}
& \frac{\partial}{\partial x_{2}} E_{p}\left(k_{1}, x_{2}, x_{3}\right) \\
& \quad=\int_{\mathbb{R}} \frac{\partial}{\partial x_{2}} V\left(y_{1}, x_{2}, x_{3}\right)\left|\phi_{p}\left(y_{1}, x_{2}, x_{3}, k_{1}\right)\right|^{2} d y_{1}
\end{aligned}
$$

Let us denote by $\Pi_{p}\left(x_{2}, x_{3}\right)$ the projectors from $L^{2}\left(\mathbb{R}^{3}\right)$ on $V_{p}\left(x_{2}, x_{3}\right)$. Let us define the operator

$$
\begin{aligned}
G_{k}= & -\frac{1}{2}\left(\frac{\partial}{\partial y_{1}}+i k_{1}\right)^{2} \cdot+V\left(y_{1}, x_{2}, x_{3}\right) . \\
= & \mathrm{e}^{i k_{1} \cdot y_{1}} H_{k}\left(\mathrm{e}^{-i k_{1_{1}}} .\right), \\
D G_{k}= & \left\{\psi \in H^{2}\left(\mathbb{R}^{3}\right), \psi\left(y_{1}+\gamma, x_{2}, x_{3}\right)\right. \\
& =\psi(y), \quad \gamma \in \Gamma\} .
\end{aligned}
$$

Therefore the projectors $\Pi_{p}\left(k_{1}, x_{2}, x_{3}\right), P_{p}\left(k_{1}, x_{2}, x_{3}\right)$ on the eigenspace corresponding to $E_{p}\left(k_{1}, x_{2}, x_{3}\right)$ for the operator $H_{k}, G_{k}$ can be defined by

$$
\begin{aligned}
& P_{p}\left(k_{1}, x_{2}, x_{3}\right) \\
& =\frac{1}{2 i \pi} \int_{|\zeta|=r}\left(G_{k}-E_{p},\left(k_{1}, x_{2}, x_{3}\right)-\zeta\right)^{-1} d \zeta \\
& \quad \Pi_{p}\left(k_{1}, x_{2}, x_{3}\right)=\mathrm{e}^{i k_{1} \cdot y_{1}} P_{p}\left(k_{1}, x_{2}, x_{3}\right)\left(\mathrm{e}^{i k_{1}} .\right)
\end{aligned}
$$

Remark 2.7 The functions $E_{p}\left(., x_{2}, x_{3}\right), P_{p}\left(., x_{2}, x_{3}\right)$ and $\Pi_{p}\left(., x_{2}, x_{3}\right)$ are analytical since we suppose 
that the energy bands $E_{p}\left(., x_{2}, x_{3}\right)$ never cross for every $p \geq 0$.

Remark 2.8 Of course we have for $\psi \in L^{2}\left(\mathbb{R}^{3}\right)$

$$
\Pi_{p}\left(x_{2}, x_{3}\right) \psi=\int_{-\pi}^{\pi} \Pi_{p}\left(k_{1}, x_{2}, x_{3}\right) \phi_{p}\left(k_{1}, x_{2}, x_{3}\right) \frac{d k_{1}}{2 \pi}
$$

where $\phi_{p}$ is the Bloch wave associated to $\psi$ and Eq. (2.9) implies that $\Pi_{p}$ commutes with $H$. More exactly we have

$$
\begin{aligned}
H \Pi_{p} \psi\left(y_{1}, x_{2}, x_{3}\right)= & \Pi_{p} H \psi\left(y_{1}, x_{2}, x_{3}\right) \\
= & \sum_{\gamma \in \mathbb{Z}} \hat{E}_{p}\left(\gamma, x_{2}, x_{3}\right) \\
& \Pi_{p} \psi\left(y_{1}+\gamma, x_{2}, x_{3}\right)
\end{aligned}
$$

Returning to the microscopic variable $y_{1}=\left(x_{1} / \varepsilon\right)$ Eq. (2.12) becomes

$$
\begin{array}{r}
{\left[-\frac{\varepsilon^{2}}{2} \cdot \frac{\partial^{2}}{\partial x_{1}^{2}}+\right.} \\
=\sum_{\gamma \in \mathbb{Z}} \hat{E}_{p}\left(\gamma, x_{2}, x_{3}\right) \\
\Pi_{p} \psi\left(x_{1}+\varepsilon \gamma, x_{2}, x_{3}\right)
\end{array}
$$

which is a "diagonalisation" of $H$ defined in (2.1).

We now turn back to Eq. (1.7), by taking the projection of (1.7) on $V_{p}$ we get

$$
i \varepsilon \partial_{t} \Pi_{P} \psi=H \Pi_{p} \psi-\frac{\varepsilon^{2}}{2} \Pi_{p}\left(\Delta_{x_{2}, x_{3}} \psi\right)
$$

where $H$ is the Hamiltonian defined in (2.1) and $\Delta_{x_{2}, x_{3}}=\left(\partial^{2} / \partial x_{2}^{2}\right)+\left(\partial^{2} / \partial x_{3}^{2}\right)$ is the Laplacian in both variable $x_{2}$ and $x_{3}$.

\section{WIGNER TRANSFORMS}

In this paper since we have periodicity in the direction $x_{1}$, we will associate Wigner series in the direction $x_{1}$, and classical Wigner transforms in the direction $x_{2}, x_{3}$.
For a couple of functions $\psi, \varphi \in L^{2}\left(\mathbb{R}^{3}\right)$, let us define

$$
\begin{gathered}
W^{\varepsilon}(\psi, \varphi)(x, k) \\
=\frac{1}{2 \pi} \int_{\mathbb{R}^{2}} \sum_{\gamma \in \mathbb{Z}} \psi\left(x_{1}-\frac{\varepsilon \gamma}{2}, x_{2}-\frac{\varepsilon z_{2}}{2}, x_{3}-\frac{\varepsilon z_{3}}{2}\right) . \\
\bar{\varphi}\left(x_{1}+\frac{\varepsilon \gamma}{2}, x_{2}+\frac{\varepsilon z_{2}}{2}, x_{3}+\frac{\varepsilon z_{3}}{2}\right) \\
\times \mathrm{e}^{i k_{1} \cdot \gamma} \mathrm{e}^{i\left(k_{2} z_{2}+k_{3} z_{3}\right)} d z_{2} d z_{3} .
\end{gathered}
$$

We define as in $[7,8,10]$ an algebra of test functions by

$$
\begin{aligned}
& \mathcal{A}=\{ w=w(x, k)=\frac{1}{(2 \pi)^{3}} \\
& \sum_{\gamma \in \mathbb{Z}} \mathrm{e}^{i k_{1} \cdot \gamma} \int_{\mathbb{R}^{2}} \hat{w}^{k_{2}, k_{3}}\left(x, \gamma, \eta_{2}, \eta_{3}\right) \mathrm{e}^{i\left(k_{2} \cdot \eta_{2}+k_{3} \cdot \eta_{3}\right)} d \eta_{2} d \eta_{3} ; \\
&\left.\hat{w} \in l^{1}\left(\mathbb{Z} ; L^{1}\left(d \eta_{2} \times \eta_{3} ; \mathcal{C}^{0}\left(\mathbb{R}_{x}^{3}\right)\right)\right)\right\}
\end{aligned}
$$

$\mathcal{A}$ is equipped with its natural norm:

$$
\begin{gathered}
\|w\|_{\mathcal{A}}=\sum_{\gamma \in \mathbb{Z}}\|\| \hat{w}^{k_{1}, k_{2}}\left(., \gamma, \eta_{2}, \eta_{3}\right) \\
\left\|_{L^{\infty}\left(\mathbb{R}^{3}\right)}\right\|_{L^{1}\left(d \eta_{2} \times d \eta_{3}\right)}
\end{gathered}
$$

Let us recall a theorem from $[7,10,6]$ :

THEOREM 3.1 Let $\left(\psi^{\varepsilon}\right)$ be a bounded family of $L^{2}\left(\mathbb{R}^{3}\right)$; then the family $\left(W^{\varepsilon}\right)=W^{\varepsilon}\left(\psi^{\varepsilon}, \psi^{\varepsilon}\right)$ is bounded in $\mathcal{A}^{\prime}$. The accumulation points in $\mathcal{A}^{\prime}$ weak - * when $\varepsilon \rightarrow 0$ are bounded non negative measures.

$$
W^{\varepsilon} \rightarrow f \quad \text { in } \quad \mathcal{A}^{\prime} \text { weak }-* .
$$

Moreover we have

$$
\begin{aligned}
& \left|\psi^{\varepsilon}\right|^{2} \rightarrow \int_{[-\pi, \pi] \times \mathbb{R}^{2}} f(., k) \frac{d k}{2 \pi} \\
& \quad \text { in } \mathcal{C}^{0^{\prime}}\left(\mathbb{R}^{3}\right) \text { weak }-*
\end{aligned}
$$

if $\left(\psi^{\varepsilon}\right)$ is $\varepsilon$-oscillatory for the variable $x_{2}, x_{3}$.

Let us now mention a property which will be very useful in the following of this paper. This is an 
expansion of Wigner transform with a pseudo differential operator $p(x, \varepsilon D)$.

Definition 3.2 Let us consider a symbol

$$
p(x, \xi) \in \mathcal{S}\left(\mathbb{R}_{x}^{m} \times \mathbb{R}_{\xi}^{m}\right)
$$

then for $f \in \mathcal{S}^{\prime}(\mathbb{R})^{m}$

$$
\begin{aligned}
& P(x, \varepsilon D) f(x) \\
& \quad=\frac{1}{(2 \pi)^{m}} \int_{\mathbb{R}^{m} \times \mathbb{R}^{m}} p(x, \varepsilon \xi) f(y) \mathrm{e}^{i(x-y) \cdot \xi} d \xi d y
\end{aligned}
$$

Definition 3.3 The Weyl operator associated to the symbol $p(x, \xi)$ is defined by

$$
\begin{aligned}
& P^{W}(x, \varepsilon D) f(x) \\
& \quad=\frac{1}{(2 \pi)^{m}} \int_{\mathbb{R}^{m} \times \mathbb{R}^{m}} p\left(\frac{x+y}{2}, \varepsilon \xi\right) f(y) \mathrm{e}^{i(x-y) \cdot \xi} d \xi d y
\end{aligned}
$$

DeFINITION 3.4 If the notation $\{p, q\}$ denotes the Poisson bracket of $p=p(x, \varepsilon)$ and $q=q(x, \xi)$ defined by

Remark 3.5 We can weaken the regularity of $p$ if we take a more regular function $f$.

$$
\begin{aligned}
\{p, q\} \quad(x, \xi)= & \nabla_{\xi} p(x, \xi) \cdot \nabla_{x} q(x, \xi) \\
& -\nabla_{x} p(x, \xi) \cdot \nabla_{\xi} q(x, \xi)
\end{aligned}
$$

Definition 3.6 Classical Wigner transform expansion with a pseudo differential operator. The classical Wigner transform of $(f, g) \in L^{2}(\mathbb{R})^{m}$ is defined by

$$
\begin{array}{rl}
w_{\varepsilon}(f, g)=\frac{1}{2 \pi^{m}} \int_{\mathbb{R}^{m}} & f\left(x-\frac{\varepsilon z}{2}\right) \\
\bar{g}\left(x+\frac{\varepsilon z}{2}\right) \mathrm{e}^{i \xi \cdot z} d z
\end{array}
$$

Property 3.6 Let $p \in \mathcal{C}^{\infty}\left(\mathbb{R}^{m} \times \mathbb{R}^{m}\right)$ satisfy for some $M \geq 0$ :

$$
\begin{aligned}
\forall \alpha \in & \left(\mathbb{N}^{m} \times \mathbb{N}^{m}\right):\left|\partial_{x, \xi}^{\alpha} \quad p(x, \xi)\right| \\
& \leq C_{\alpha}(1+|\xi|)^{M} .
\end{aligned}
$$

If $f$ and $y$ lie in a bounded set of $L^{2}(\mathbb{R})^{m}$, we have the expansion

$$
\begin{aligned}
w_{\varepsilon}\left(P^{W}(x, \varepsilon D) f, g\right)= & p w_{\varepsilon}(f, g) \\
& +\frac{\varepsilon}{2 i}\left\{p(x, \xi), w_{\varepsilon}(f, g)\right\} \\
& +\varepsilon^{2} r_{\varepsilon}
\end{aligned}
$$

where $r_{\varepsilon}$ is bounded in $\mathcal{S}^{\prime}\left(\mathbb{R}^{m} \times \mathbb{R}^{m}\right)$ uniformly w.r.t. $\varepsilon$.

This property is proved in [6].

Theorem 3.7 Let $p \in \mathcal{C}^{\infty}\left(\mathbb{R}^{2} \times \mathbb{R}^{2}\right)$ satisfy for some $M \geq 0$,

$$
\begin{array}{r}
\forall \alpha \in\left(\mathbb{N}^{2} \times \mathbb{N}^{2}\right):\left|\partial_{x_{2}, x_{3}, k_{2}, k_{3}}^{\alpha} p\left(x_{2}, x_{3}, k_{2}, k_{3}\right)\right| \\
<C_{\alpha}(1+|\xi|)^{M}
\end{array}
$$

Then as $\phi$ and $\varphi$ lie in a bounded set of $L^{2}\left(\mathbb{R}^{3}\right)$ we have the expansion

$$
\begin{aligned}
W^{\varepsilon}\left(P^{W}\left(x_{2}, x_{3}, \varepsilon D_{2,3}\right) \psi, \varphi\right) & \\
= & p\left(x_{2}, x_{3}, k_{2}, k_{3}\right) W^{\varepsilon}(\psi, \varphi) \\
& +\frac{\varepsilon}{2 i}\left\{p\left(x_{2}, x_{3}, k_{2}, k_{3}\right), W^{\varepsilon}(\psi, \varphi)\right\}+\varepsilon^{2} R_{\varepsilon}
\end{aligned}
$$

where $R_{\varepsilon}$ is bounded in $\mathcal{S}^{\prime}(\mathbb{R})^{6}$ uniformly w.r.t. $\varepsilon$

Theorem 3.8 Let $a \in \mathcal{C}^{0}\left(\mathbb{R}^{2} x_{2}, x_{3}, \mathcal{C}^{1}\left(\mathbb{R}_{k_{1}}\right)\right)$ 1-periodic with respect to $k_{1}$. Then as $\psi$ and $\varphi$ lie in a bounded set of $L^{2}(\mathbb{R})^{3}$ we have the expansion

$$
\begin{aligned}
W^{\varepsilon}( & \left.a\left(\varepsilon D_{1}, x_{2}, x_{3}\right) \psi, \varphi\right) \\
= & \left(a\left(D_{1}, x_{2}, x_{3}\right) W^{\varepsilon}(\psi, \varphi)\right. \\
& -\frac{\varepsilon}{2 i} \nabla_{x_{2}, x_{3}} a\left(k_{1}, x_{2}, x_{3}\right) \cdot \nabla_{k_{2}, k_{3}} W^{\varepsilon}(\psi, \varphi) \\
& +\frac{\varepsilon}{2 i} \nabla_{k_{1}} a\left(k_{1}, x_{2}, x_{3}\right) \cdot \nabla_{x_{1}} W^{\varepsilon}(\psi, \varphi)+\varepsilon^{2} T_{\varepsilon} .
\end{aligned}
$$

in $\mathcal{S}^{\prime}$ with $T_{\varepsilon}$ bounded in $\mathcal{S}^{\prime}\left(\mathbb{R}^{6}\right)$ uniformly w.r.t. $\varepsilon$. 
The proof of Theorem (3.7) and Theorem (3.8) is almost the same as in [6]: we make a Taylor expansion of the Fourier transform of the Wigner function and we get the desired result.

\section{COMMUTATOR ESTIMATES}

Let $\psi_{\varepsilon}$ be a bounded family of functions in $L^{2}(\mathbb{R})^{3}$ satisfying Eq. (1.7). $\Pi_{p} \psi$ satisfies Eq. (2.14) with $\Pi_{p} \psi_{I \varepsilon}=\psi_{I \varepsilon} \in V_{p}$. In order to pass to the limit $\varepsilon \rightarrow 0$, let us rewrite Eq. (2.14) in an other way:

$$
\begin{aligned}
i \varepsilon \partial_{t} \Pi_{p} \psi= & i E_{p}\left(\varepsilon D_{1}, x_{2}, x_{3}\right) \Pi_{p} \psi \\
& -\frac{\varepsilon^{2}}{2} \Delta_{x_{2}, x_{3}} \Pi_{p} \psi+\frac{\varepsilon^{2}}{2}\left[\Delta_{x_{2}, x_{3}} ; \Pi_{p}\right] \psi
\end{aligned}
$$

where $\left[\Delta_{x_{2}, x_{3}} ; \Pi_{p}\right]$ is a commutator defined by

$$
\left[\Delta_{x_{2}, x_{3}} ; \Pi_{p}\right] \psi=\Delta_{x_{2}, x_{3}} \Pi_{p} \psi-\Pi_{p} \Delta_{x_{2}, x_{3}} \psi
$$

\section{Proposition 4.1}

$$
\begin{aligned}
& \frac{1}{\varepsilon}\left(W^{\varepsilon}\left(\left[\Delta_{x_{2}, x_{3}} ; \Pi_{p}\right] \psi, \Pi_{p} \psi\right)\right. \\
& \left.\quad-W^{\varepsilon}\left(\Pi_{p} \psi,\left[\Delta_{x_{2}, x_{3}} ; \Pi_{p} \psi\right]\right)\right) \rightarrow 0 \\
& \quad \text { in } L^{\infty}\left(0, T ; \mathcal{S}^{\prime}\left(\mathbb{R}^{6}\right)\right)
\end{aligned}
$$

follows from these very useful lemmas

LemmA 4.1 Let $\psi_{p}^{\varepsilon} \in V_{p}^{\varepsilon}, \psi_{q}^{\varepsilon} \in V_{q}^{\varepsilon}$ be two bounded sequences in $L^{2}\left(\mathbb{R}^{3}\right)$ satisfying the equations

$$
\begin{aligned}
& H \psi_{p}^{\varepsilon}=E_{p}\left(\varepsilon D_{1}, x_{2}, x_{3}\right) \psi_{p}^{\varepsilon} \\
& H \psi_{q}^{\varepsilon}=E_{q}\left(\varepsilon D_{1}, x_{2}, x_{3}\right) \psi_{q}^{\varepsilon}
\end{aligned}
$$

where $H$ is the Hamiltonian defined in Eq. (2.1). We have

$$
\begin{aligned}
W^{\varepsilon}\left(\psi_{p}^{\varepsilon}, \psi_{q}^{\varepsilon}\right)= & \frac{\varepsilon^{2}}{E_{p}-E_{q}} \operatorname{div}_{x_{1}} W^{\varepsilon}\left(\partial_{x_{1}} \psi_{p}^{\varepsilon}, \psi_{q}^{\varepsilon}\right) \\
& +\varepsilon^{2}\left\|\psi_{p}^{\varepsilon}\right\|_{L^{2}}\left\|\psi_{q}^{\varepsilon}\right\|_{L^{2}} R^{\varepsilon}
\end{aligned}
$$

$$
\begin{aligned}
= & \frac{\varepsilon}{\left(E_{p}-E_{q}\right)^{2}} d i v_{x_{1}} \\
& W^{\varepsilon}\left(\partial_{x_{1}} V\left(\frac{x_{1}}{\varepsilon}, x_{2}, x_{3}\right) \psi_{p}^{\varepsilon}, \psi_{q}^{\varepsilon}\right) \\
& +\varepsilon^{2}\left\|\psi_{p}^{\varepsilon}\right\|_{L^{2}}\left\|\psi_{q}^{\varepsilon}\right\|_{L^{2}} R^{\varepsilon}
\end{aligned}
$$

where $R^{\varepsilon}$ is uniformly bounded in $\mathcal{S}^{\prime}\left(\mathbb{R}^{6}\right)$ with respect to $\varepsilon, \psi_{p}^{\varepsilon}$ and $\psi_{q}^{\varepsilon}$.

Lemma 4.2 Let $\psi^{\varepsilon}$ be a function such that $\psi^{\varepsilon}$, $H^{\varepsilon} \psi$ are bounded sequences in $L^{2}$, then

$$
\left\|\Pi_{p} \psi\right\|_{L^{2}} \leq \frac{C}{p^{2}} .
$$

Of course this implies

$$
\int_{\mathbb{R}^{3}} n_{p} d x \leq \frac{C}{c p^{4}}
$$

Therefore the sequence $\Sigma_{p} n_{p}$ is uniformly convergent w.r.t. $\varepsilon$.

Proof of Proposition (4.1) In the following, we will note with a prime' a derivation w.r.t. the variable $x_{2}$ or $x_{3}$ and define $\psi_{p}=\Pi_{p} \psi$. We have

$$
\Pi_{p} \varepsilon^{2} \psi^{\prime \prime}=\varepsilon^{2} \psi_{p}^{\prime \prime}+\sum_{q \neq p}\left(\psi_{q}^{\prime \prime}-\Pi_{q}\left(\varepsilon^{2} \psi^{\prime \prime}\right)\right)
$$

Therefore we have

$$
\begin{aligned}
W^{\varepsilon}\left(\Pi_{p} \varepsilon^{2} \psi^{\prime \prime}, \psi_{p}\right)= & W^{\varepsilon}\left(\varepsilon^{2} \psi_{p}^{\prime \prime}, \psi_{p}\right)+\sum_{q \neq p}\left(W^{\varepsilon}\left(\psi_{q}^{\prime \prime}, \psi_{p}\right)\right. \\
& \left.-W^{\varepsilon}\left(\Pi_{q}\left(\varepsilon^{2} \psi^{\prime \prime}\right), \psi_{p}\right)\right)
\end{aligned}
$$

Since the series $\Sigma_{q \neq p} \Pi_{q} \psi$ and $\Sigma_{q \neq p} \Pi_{q} \varepsilon^{2} \psi^{\prime \prime}$ converge uniformly by Lemma (4.2) see also $[6,8]$, we only have to verify that for every fixed $q$, the terms with different indices converge to zero in $\mathcal{S}^{\prime}\left(\mathbb{R}^{6}\right)$. By a little computation using Lemma (4.1) and the 
asymptotic expansion of Theorem (3.7), we have

$$
\begin{aligned}
& W^{\varepsilon}\left(\psi_{q}^{\prime \prime}, \psi_{p}\right)-W^{\varepsilon}\left(\Pi_{q}\left(\varepsilon^{2} \psi^{\prime \prime}\right), \psi_{p}\right) \\
&= \varepsilon \operatorname{div}_{x_{1}}\left[W ^ { \varepsilon } \left(\partial_{x_{1}} V\left(\frac{x_{1}}{\varepsilon}, x_{2}, x_{3}\right)\right.\right. \\
&\left.\left.\times\left(2 \varepsilon \Pi_{q}^{\prime} \varepsilon \psi^{\prime}+\varepsilon^{2} \Pi_{q}^{\prime \prime} \psi, \psi_{p}\right)\right)\right]+O\left(\varepsilon^{2}\right) \\
&= O\left(\varepsilon^{2}\right)
\end{aligned}
$$

We are done.

\section{SEMI-CLASSICAL LIMIT}

We now compute the semi-classical limit i.e., $\varepsilon \rightarrow 0$ of the Wigner equation.

THEOREM 5.1 As $\varepsilon \rightarrow 0$, if for some subsequence $W_{I}^{\varepsilon} \rightarrow f_{p I}$ in $\mathcal{A}^{\prime}$-weak ${ }^{*}$, then we have $W^{\varepsilon} \rightarrow f_{p}$ in $\mathcal{C}^{0}\left(0, T, \mathcal{S}^{\prime}(\mathbb{R})^{6}\right)$

where $f_{p} \geq 0$ verifies the Vlasov equation:

$$
\begin{gathered}
\partial_{t} f_{p}=\left\{\mathcal{E}_{p}, f_{p}\right\} \\
f_{p}(0, x, k)=f_{p I}(x, k) .
\end{gathered}
$$

where

$$
\mathcal{E}_{p}\left(k, x_{2}, x_{3}\right)=E_{p}\left(k_{1}, x_{2}, x_{3}\right)+\frac{\left|k_{2}\right|^{2}}{2}+\frac{\left|k_{3}\right|^{2}}{2}
$$

and $n^{\varepsilon}(t, x) \rightarrow n(t, x)$ in $C\left(0, T, C^{0^{\prime}}\left(\mathbb{R}^{3}\right)-\right.$ weak $\left.*\right)-$ weak* where:

$$
n(x, t)=\sum_{p} \int_{[-\pi, \pi] \times \mathbb{R}^{2}} f_{p}(x, k, t) \frac{d k}{2 \pi} .
$$

Remark 5.2 In extended form the Eq. (5.1) reads

$$
\begin{aligned}
\partial_{t} f_{p}= & \left(\begin{array}{c}
\partial_{k_{1}} E_{p}\left(k_{1}, x_{2}, x_{3}\right) \\
k_{2} \\
k_{3}
\end{array}\right) \cdot \nabla_{x} f_{p}(t, x, k) \\
& -\nabla_{x_{2}, x_{3}} E_{p}(t, x, k) \cdot \nabla_{k_{2}, k_{3}} f_{p}(t, x, k)
\end{aligned}
$$

The proof of Theorem (5.1) follows immediately from proposition (4.1) by using the asymptotic expansions from Theorems (3.7) and (3.8) applied to Eq. (4.1).

\section{References}

[1] Bechouche, P. (1997). Semi-classical Limits in a Crystal with a Coulombian self-consistent Potential: Effective Mass Theorems. to appear.

[2] Bensoussan, A., Lions, J.-L. and Papanicolaou, G. (1978). Asymptotic Analysis for Periodic Structures. North-Holland, Amsterdam-New York-Oxford.

[3] Colin de Verdiére, Y. (1991). "Sur les singularités de Van Hove génériques," in Colloque à la mémoire d'Edmond Combet. Bulletin de la SMF (119), 46.

[4] Gérard, P. (1991). Mesures semi-classiques et ondes de Bloch. Sem. Ecole Polytechnique, XVI, 1-19.

[5] Gérard, P., On the semiclassical derivation of transport equations in semiconductors. First International Conference on Open Problems in charged Particle Transport, June 1996.

[6] Gérard, P., Markowich, P., Mauser, N. and Poupaud, F. (1997). Homogenization limits and Wigner transforms. Communication on Pure and Applied Mathematics, 50, $0323-0379$.

[7] Lions, P. L. and Paul, T. (1993). Sur les mesures de Wigner. Revista Mat. Iberoamericana, 9, 553-618.

[8] Markowich, P. A., Mauser, N. J. and Poupaud, F. (1994). A Wigner function approach to semiclassical limits: electrons in a periodic potential. J. Math. Phys., 35, $1066-1094$.

[9] Poupaud, F. and Ringhofer, C. (1995). Quantum hydrodynamic models in a crystal. Applied Math. Lett., 8.

[10] Poupaud, F. and Ringhofer, C. (1996). Semi-classical Limits in a Crystal with an external potential, Effective Mass Theorems. Communication in Partial differential Equations, 21.

[11] Reed, M. and Simon, B. (1987). Methods of Modern Mathematical Physics IV. Academic Press, New York-San Francisco-London, 4th edition.

[12] Wilcox, C. H. (1978). Theory of Bloch waves. J. d'Analyse Math., 33, 146-167.

\section{Author Biography}

Philippe Bechouche is a Researcher in Mathematics at the University of Nice Sophia-Antipolis. His research interests are in modeling classical and quantum semiconductors - more specifically in how semiclassical limits describe the link between quantum equations and kinetic equations and permit us to derive classical models of semiconductors with quantum corrections. 

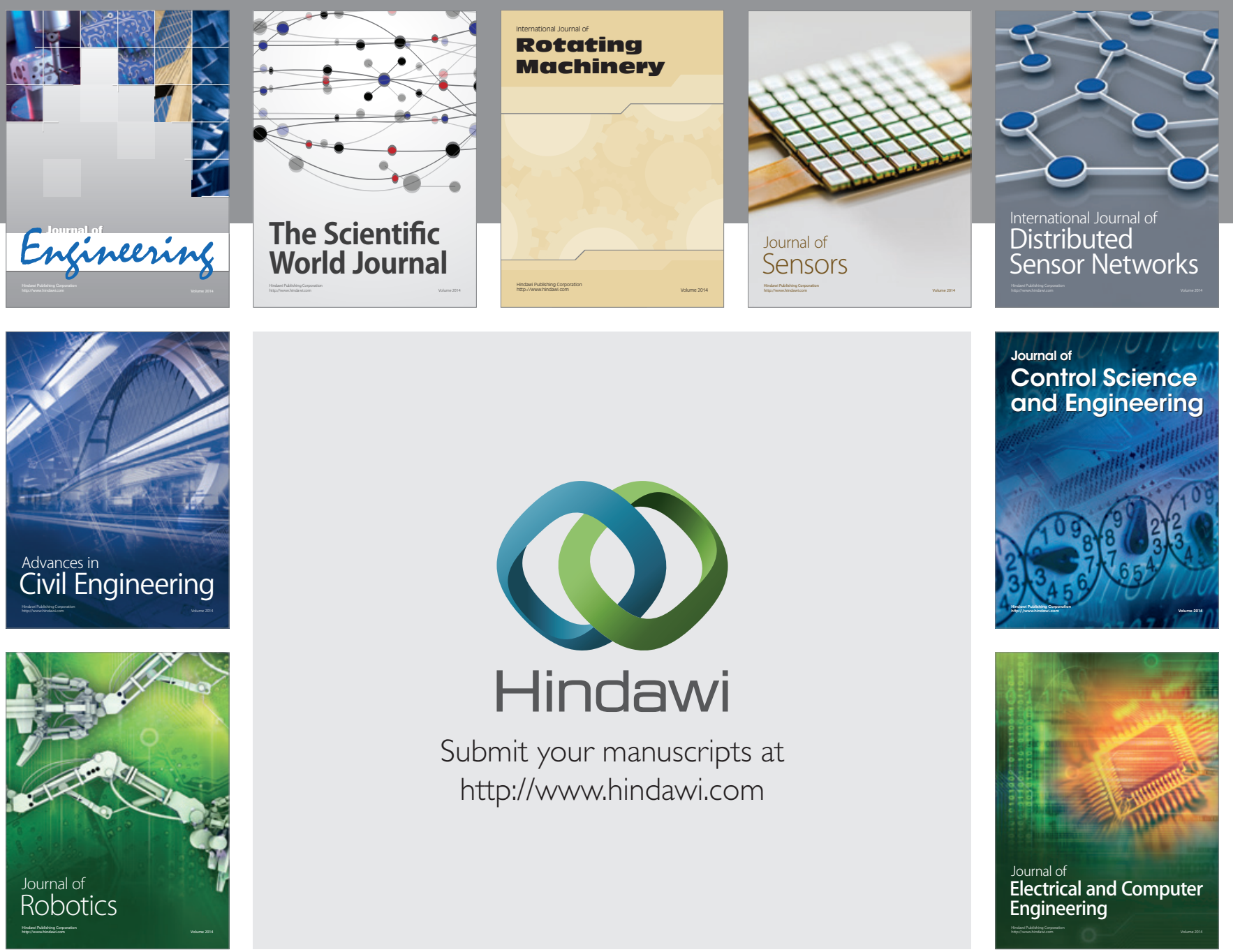

Submit your manuscripts at

http://www.hindawi.com
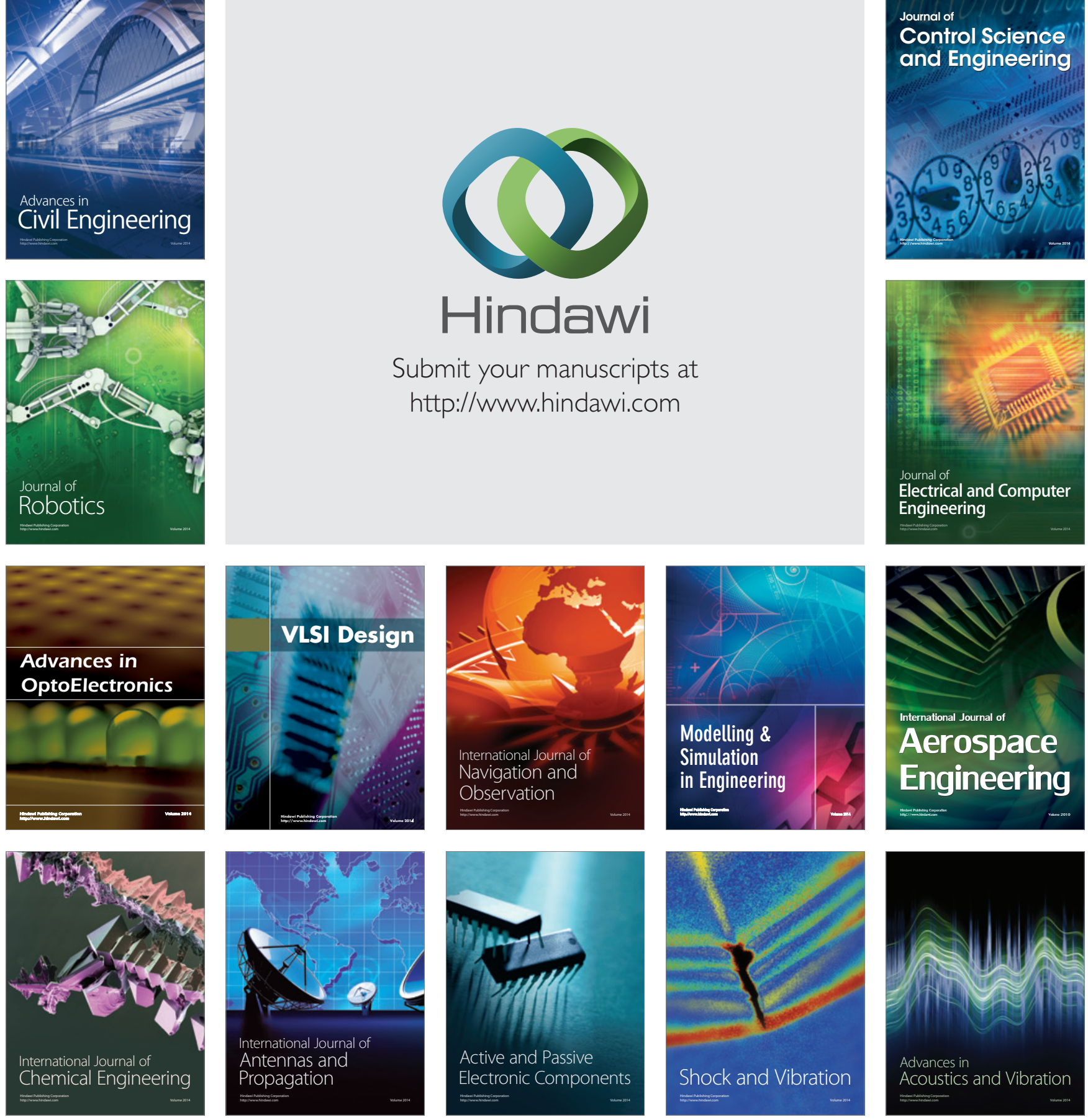